\title{
MITIGACIÓN DE LOS GASES DE EFECTO INVERNADERO EN LOS SECTORES DIFUSOS EN ESPAÑA. FINANCIACIÓN CLIMÁTICA Y POTENCIAL DE LA BIOMASA: CASO PRÁCTICO AVEBIOM
}

\author{
Autora ${ }^{1}$ : Silvia López Sánchez² \\ Responsable del Programa Canal Clima \\ Asociación Española de Valorización Energética de la Biomasa (AVEBIOM) \\ Spanish Bioenergy Association
}

\section{Resumen}

El potencial de reducción de emisiones de gases de efecto invernadero en los denominados "sectores difusos" en España, a través de acciones de mitigación basadas en medidas de eficiencia energética e implantación de energías renovables, es muy alto. Con el fin de impulsar la transformación del sistema productivo español en un modelo bajo en emisiones de gases de efecto invernadero, se han

\footnotetext{
${ }^{1}$ Deseo agradecer a María Jesús Romero Olid su estimada colaboración en la realización del presente trabajo.

${ }^{2}$ silvialopez@avebiom.org
} 
aprobado varias líneas de financiación para estos sectores. En este artículo se analiza el papel que puede jugar la biomasa en la descarbonización gradual de la matriz energética, con iniciativas como los Proyectos Clima.

Palabras clave: Mitigación; sectores difusos; eficiencia energética; energías renovables; financiación climática; FES-CO ${ }_{2}$ Proyectos Clima; reducciones verificadas de emisiones; biomasa.

GHGs mitigation in diffuse sectors: Climate funding and biomass potential in Spain and climate funding. Success story

\begin{abstract}
The potential for reducing emissions of greenhouse gases in the so-called "diffuse sectors" in Spain, through mitigation actions based on energy efficiency measures and implementation of renewable energy is extraordinarily high. In order to promote the transformation of the Spanish production system into a low emissions of greenhouse gases model, several funding lines have been approved for these sectors. This article describes the role that biomass can play in the gradual decarbonization of the energy matrix, with initiatives such as the Climate Projects.
\end{abstract}

Key words: Mitigation; diffuse sectors; energy efficiency; renewables energies; climate funding; FES-CO2; Climate Projects; verified emissions reduction emissions; biomass

\title{
1. MITIGACIÓN DE LOS GASES DE EFECTO INVERNADERO EN LOS SECTORES DIFUSOS
}

La mitigación es la intervención humana para reducir los efectos potenciales del calentamiento global causados por la generación de gases de efecto invernadero, lo que implica la reducción de estos gases ya sea evitando su generación, mediante la reducción de sus fuentes, o aumentando su captura o almacenamiento.

La dinámica de la vegetación y del suelo puede influir en la captación y retención del carbono a la atmósfera. Por ejemplo, en España, como sumidero, el uso 
del suelo, los cambios en el uso del suelo y el sector forestal fluctúa entre 29.000 y $30.000 \mathrm{tCO}_{2}$ eq anuales ${ }^{3}$.

La captación y almacenamiento de $\mathrm{CO}_{2}$ puede aplicarse, aunque no es objeto de estudio en este artículo, a grandes fuentes puntuales para ser almacenado en formaciones geológicas, en el océano, en carbonatos minerales, o para ser utilizado en procesos industriales. La finalidad de la captación de $\mathrm{CO}_{2}$ es producir un flujo concentrado de $\mathrm{CO}_{2}$ que, a alta presión, pueda ser fácilmente transportado a su lugar de almacenamiento. Si bien, en principio, la totalidad del flujo de gas con bajas concentraciones de $\mathrm{CO}_{2}$ podría ser transportada e inyectada bajo tierra, por lo general, el coste de la energía necesaria en el proceso y otros gastos asociados hacen que este método sea poco práctico y difícilmente viable económicamente ${ }^{4}$.

Por esta razón, en este artículo se van a abordar otras opciones de mitigación que también se puede utilizar y que evitan o reducen las emisiones en su fuente. El caso más claro es sustituir directamente el empleo de combustibles contaminantes por otras fuentes de energía renovable.

En España, más del 50\% de las emisiones se deben al sector transporte y al residencial, sectores que no están cubiertos por la Directiva 96/61/CE, de 24 de septiembre de 1996, relativa a la prevención y al control integrados de la contaminación $(\text { IPPC })^{5}$. Las emisiones del transporte han crecido vertiginosamente en los últimos años (casi un 5\% anual) y son, junto con las del sector residencial, las que más pueden dificultar el cumplimiento de los compromisos asumidos en virtud de la Decisión de la Comisión Europea 2013/162/UE de 26 de marzo de 2013, por la que se determinan las asignaciones anuales de emisiones de los Estados miembros para el período de 2013 a 2020, de conformidad con la Decisión 406/2009/CE del Parlamento Europeo y del Consejo ${ }^{6}$.

Según esta Decisión, España en el año 2020 debe cumplir el objetivo de reducir las emisiones de $\mathrm{CO}_{2}$ en los denominados sectores difusos ${ }^{7}$, en un $10 \%$ con respecto al año 2005. Esto significa evitar la generación de aproximadamente, 2 millones de toneladas de $\mathrm{CO}_{2}$ al año.

\footnotetext{
${ }^{3}$ Ver Inventarios Nacionales de Emisiones.

${ }^{4}$ Esta opción de mitigación, se aborda ampliamente en el estudio publicado en 2005, Informe especial del IPCC. La captación y el almacenamiento de dióxido de carbono, preparado por el Grupo Integubernamental de Expertos de Cambio Climático.

${ }^{5}$ DO L 257 de 10.10.1996. Actualizada en la Directiva 2008/1/ce del parlamento europeo y del consejo de 15 de enero de 2008 relativa a la prevención y al control integrados de la contaminación DO L 24/8 a 24/29 de 29.1.2008 (Versión codificada).

6 "Effort Sharing Decision", DECISIÓN 406/2009/CE del Parlamento Europeo y del Consejo de 23 de abril de 2009 sobre el esfuerzo de los estados miembros para reducir sus emisiones de gases de efecto invernadero.

${ }^{7}$ Residencial, transporte, agrícola y ganadero, residuos, gases fluorados y la industria no sujeta al comercio de derechos de emisión.
} 
Gráfico 1. Límites de gases de efecto invernadero para los Estados Miembros en el año 2020, comparados con los niveles de 2005

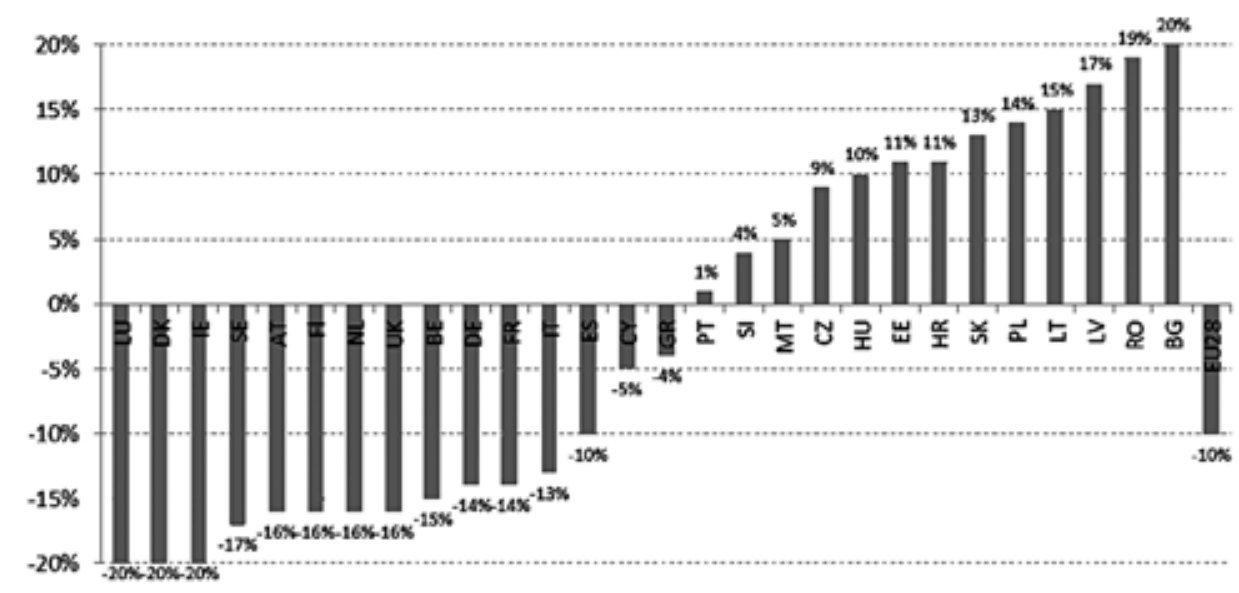

Fuente: Comisión Europea: http://ec.europa.eu/clima/policies/effort/index_en.htm

Esta meta, junto a otros indicadores objetivo identificados para España en la Estrategia Europea 2020 (participación de las fuentes renovables en nuestro consumo final de energía en un 20\% y la mejora de la eficiencia energética en un 20\%), marcan claramente la "hoja de ruta" a seguir en actuaciones de mitigación.

El sector residencial, comercial y de servicios no participa en los mercados de carbono ni tampoco la pequeña y mediana industria e instalaciones agroganaderas. Este mercado está reservado a las grandes instalaciones consumidoras de grandes cantidades de energía y, por tanto, generadoras de un gran volumen de emisiones; sin embargo, la actuación en los sectores difusos mediante ayudas e incentivos financieros adecuados, tiene un enorme potencial para mejorar tanto su eficiencia energética y el rendimiento de sus instalaciones, como para reducir el consumo de combustibles fósiles, empleando fuentes de energía renovables.

Existe un caso que muestra las ventajas que puede tener directa a indirectamente una adecuada actuación en los sectores difusos: el sector de la rehabilitación inmobiliaria en España. La mayoría del parque inmobiliario español, con cerca de 25 millones de viviendas, está construido sin criterios de eficiencia energética, dado que el $90 \%$ de estos edificios son anteriores a la aplicación del Real Decreto 314/2006, de 17 de marzo, por el que se aprueba el Código Técnico de la Edificación ${ }^{8}$ y el $60 \%$ de las viviendas españolas se construyó antes de que hubiera

\footnotetext{
${ }^{8}$ BOE núm. 74, de 28 de marzo de 2006, páginas 11816 a 11831.
} 
una normativa que regulara este aspecto 9 . Según se expone en este Real Decreto $314 / 2006^{10}$, se puede proceder a una rehabilitación de los edificios que permita una mayor eficiencia energética, más uso de energías renovables y, además de los efectos positivos desde el punto de vista medioambiental, se podrían tener efectos muy beneficiosos a nivel económico dada la generación de empleo y renta que siempre lleva asociado el sector inmobiliario.

\section{FINANCIACIÓN DE ACTUACIONES DE MITIGACIÓN QUE REDUZCAN O EVITEN EMISIONES}

Además de mejorar la eficiencia energética, las medidas más contundentes para lograr mitigar el cambio climático pasan por un aumento en el empleo de energías renovables.

Ambos aspectos se están beneficiando en los últimos años de líneas de financiación específicas que estimulan la inversión sostenible y la "descarbonización" de estos llamados "sectores difusos".

Aunque se podrían destacar diversos programas puestos en marcha en los últimos años en España, entre los más recientes se pueden citar:

1. Programa de Ayudas para la rehabilitación energética de los edificios existentes del sector residencial (Programa PAREER CRECE), del Instituto para la Diversificación y Ahorro de la Energía de Desarrollo (IDAE),

2. Programa de Ayudas para actuaciones de eficiencia energética en Pyme y en gran empresa del sector industrial, del Ministerio de Industria, Energía y Turismo ${ }^{11,} \mathrm{y}$,

3. Línea de financiación climática del FES- $\mathrm{CO}_{2}$, del Ministerio de Alimentación, Agricultura y Medio Ambiente. Proyectos Clima.

${ }^{9}$ Ver Instituto de Desarrollo y Ahorro Energético, IDAE.

${ }^{10}$ El Grupo de Trabajo sobre Rehabilitación (GTR) es un grupo formado como un instrumento de continuación del trabajo de diversos congresos desarrollados durante el año 2010: Conferencia Internacional Sustainable Building (SB10Mad), en Madrid; Rehabilitación y sostenibilidad. El futuro es posible $(\mathrm{R}+\mathrm{S}=\mathrm{F})$, en Barcelona); Décimo Congreso Nacional del Medio Ambiente (Conama10) en Madrid. En todos ellos se destacó la necesidad de proponer un cambio en el sector de la edificación para abordar los retos que debe afrontar la economía española entre ellos, muy especialmente, los retos ambientales.

En este sector de la edificación actualmente tiene un papel fundamental la rehabilitación lo que explica este Grupo de Trabajo, coordinado por Green Building Council España, y su interés en colaborar con otras entidades, en concreto, la Fundación CONAMA

Véase http://www.gbce.es/archivos/ckfinderfiles/GTR/Informe\%20GTR\%202014.pdf

${ }^{11}$ Resolución de 28 de abril de 2015, del Instituto para la Diversificación y Ahorro de la Energía, por la que se publica la Resolución de 24 de marzo de 2015, del Consejo de Administración, por la que se establecen las bases reguladoras y convocatoria del programa de ayudas para actuaciones de eficiencia energética en PYME y en gran empresa del sector industrial. 


\subsection{Programa de Ayudas para la rehabilitación energética de los edificios existentes del sector residencial. Programa PAREER -CRECE}

En la Resolución de 25 de junio de 2013, del Consejo de Administración del Instituto para la diversificación y ahorro de la Energía, publicada mediante la Resolución de 25 de septiembre de 2013 de la Secretaría de Estado de Energía ${ }^{12}$, se establecieron las bases reguladoras y la convocatoria del Programa de Ayudas para la Rehabilitación Energética de Edificios Existentes del sector Residencial (uso vivienda y hotelero) (Programa PAREER).

Este programa se desarrollaba dentro del contexto de lo estipulado por el art. 4 de la Directiva 2012/27/UE, relativa a la eficiencia energética, en la que se obligaba a los Estados miembros de la UE a desarrollar una estrategia a largo plazo para incentivar inversiones en la "renovación exhaustiva y rentable de edificios residenciales y comerciales, con el fin de mejorar el rendimiento energético del parque inmobiliario y reducir su consumo de energía".

Este Programa PAREER se empezó a aplicar el 2 de octubre de 2013; sin embargo, su evolución y la aprobación del Plan de Medidas para el Crecimiento, la Competitividad y la Eficiencia (CRECE) ${ }^{13}$, exigió un reajuste del programa que se realizó en la Resolución de 28 de abril de 2015, del Instituto para la Diversificación y el Ahorro de la Energía, por la que se publica la Resolución de 24 de marzo de 2015, del Consejo de Administración, por la que se modifican las bases reguladoras y convocatoria del programa de ayudas para la rehabilitación energética de edificios existentes del sector residencial (uso vivienda y hotelero) publicada en el BOE de 5 de mayo de $2015^{14}$.

A partir de este momento, al programa se le denomina Programa de ayudas para la rehabilitación energética de edificios existentes (Programa PAREER-CRE$\mathrm{CE}^{15}$ ) lo que refleja mejor la ampliación de su objeto a todas aquellas actuaciones de reforma que favorezcan el ahorro, mejora de la eficiencia energética y reducción de emisiones de $\mathrm{CO}_{2}$ en los edificios existentes, sin limitar su ámbito de aplicación al sector residencial ni a su uso como vivienda u hotel, incluyendo así expresa mención relativa a la nueva financiación proveniente del Plan CRECE. Además, como se había puesto ya en marcha el Programa Operativo Plurirregional de Crecimiento Sostenible para la aplicación de los fondos FEDER correspondientes al periodo 2014-202016, se establecieron unas condiciones adicionales para posibilitar que las ayudas objeto del Programa pudieran ser cofinanciadas con fondos comunitarios.

\footnotetext{
${ }^{12}$ Boletín Oficial del Estado número 235, de 1 de octubre de 2013.

${ }^{13}$ Cuya puesta en marcha se aprobó en Consejo de Ministros de 6 de junio de 2014.

${ }^{14}$ Boletín Oficial del Estado número 107, de 5 de mayo de 2015, pp.39354-39398.

${ }^{15} \mathrm{http}: / /$ www.idae.es/

${ }^{16}$ Se puede consultar http://www.dgfc.sgpg.meh.es/
} 
El Programa PAREER - CRECE, cuenta con un presupuesto total de $200 \mathrm{mi}$ llones de euros ${ }^{17}$ y es un Programa combinado de ayudas sin contraprestación (hasta un 35\% sobre coste elegible) y con préstamo reembolsable que completa la ayuda hasta un $90 \%$ del total de los costes elegibles si se trata de particulares y empresas y hasta un $100 \%$ si son Administraciones Públicas.

Los fondos recibidos deben destinarse a los edificios existentes, independientemente de su uso, que acometan actuaciones en envolvente térmica; actuaciones de eficiencia energética incluyendo iluminación; o implantación de biomasa, energía solar o geotérmica para consumos térmicos que reemplacen combustible fósil. En todos los casos, es un requisito imprescindible mejorar al menos 1 letra medida en la escala de emisiones de dióxido de carbono ( $\mathrm{kg} \mathrm{CO} 2 / \mathrm{m} 2$ año), con respecto a la calificación energética inicial del edificio, lo que se verificará mediante el certificado de eficiencia energética que debe realizarse antes y después de haber llevado a cabo la actuación.

\subsection{Programa de Ayudas para actuaciones de eficiencia energética en Pyme y en gran empresa del sector industrial, del Ministerio de Industria, Energía y Turismo}

Este Programa se ha centrado en actuaciones de eficiencia energética en Pyme y gran empresa del sector industrial ${ }^{18}$, consistente en ayudas a fondo perdido de hasta el 30\%, con un máximo por solicitud de cuatro millones de euros. Todas las actuaciones deben ir destinadas a: fomentar el uso de tecnologías de alta eficiencia energética o mejorar la tecnología disponible; implantar los procesos industriales y sistemas de gestión energética que tengan como objetivo reducir el consumo energético y las emisiones de $\mathrm{CO}_{2}$.

Inicialmente, la dotación presupuestaria de este Programa de Ayudas era de 49.016.421 euros, con origen en el Fondo Nacional de Eficiencia Energética, creado por la Ley 18/2014, de 15 de octubre, de aprobación de medidas urgentes para el crecimiento, la competitividad y la eficiencia ${ }^{19}$, no obstante, debido al éxito de esta convocatoria, se ha ampliado esta dotación con 66 millones de euros más.

\subsection{Línea de financiación climática del FES-CO2, del Ministerio de Alimentación, Agricultura y Medio Ambiente. Proyectos Clima ${ }^{20}$}

La línea de financiación del Fondo de Carbono para una Economía Sostenible (FES-CO ${ }_{2}$ ) se creó en el año 2011 a iniciativa del Ministerio de Agricultura,

\footnotetext{
${ }^{17}$ Ver http://www.idae.es/index.php/id.858/relmenu.409/mod.pags/mem.detalle

${ }^{18} \mathrm{http} / / /$ www.idae.es/index.php/relcategoria.4037/id.856/relmenu.454/mod.pags/mem.detalle

${ }^{19}$ BOE núm. 252, de 17 de octubre de 2014, pp. 83921 a 84082.

${ }^{20} \mathrm{http} / /$ www.magrama.gob.es/es/cambio-climatico/temas/proyectos-clima/
} 
Alimentación y Medio Ambiente y adscrito a la Secretaría de Estado de Cambio Climático ${ }^{21}$ para la compra de créditos de carbono.

El fondo está concebido como un nuevo instrumento de financiación para reorientar la actividad económica hacia modelos bajos en carbono, al mismo tiempo que contribuye al cumplimiento de los objetivos internaciones asumidos por España en materia de reducción de gases de efecto invernadero. Se destina a dos tipos de actuaciones:

- operaciones de adquisición de créditos internacionales,

- operaciones que afectan a reducciones verificadas de emisiones derivadas de proyectos ubicados en el territorio nacional, englobados en la categoría de Proyectos Clima.

Los Proyectos Clima deberán cumplir una serie de requisitos claramente definidos en el art. 7 del Real Decreto 1494/2011:

- Su ejecución no deberá venir exigida por la normativa sectorial que les resulte de aplicación.

- Deberán contribuir al cumplimiento de los compromisos cuantificados de limitación o reducción de emisiones asumidos por España mediante el logro de reducciones que tengan reflejo en el Inventario de Gases de Efecto Invernadero.

- Las reducciones de emisiones de gases de efecto invernadero que generen no procederán de instalaciones sujetas al régimen de comercio de derechos de emisión, con el objeto de evitar supuestos de doble contabilidad.

- No podrán en ningún momento ser reconocidos como proyectos de aplicación conjunta.

Los Proyectos Clima priman la adquisición de reducciones verificadas de emisiones en los conocidos como "sectores difusos" no sujetos al Régimen Europeo de Comercio de Derechos de Emisión. Mediante la adquisición de estos créditos de carbono ${ }^{22}$, el FES- $\mathrm{CO}_{2}$ moviliza recursos y elimina barreras a la inversión, fomentando el uso de tecnologías limpias, energías renovables y medidas de eficiencia energética.

A grandes rasgos los Proyectos Clima se caracterizan por ser:

${ }^{21}$ Real Decreto 1494/2011, de 24 de octubre, por el que se regula el Fondo de Carbono para una Economía Sostenible. BOE núm. 270, de 9 de noviembre de 2011, pp. 116658 a 116666.

${ }^{22}$ El precio de compra fijado en la Convocatoria 2015 fue de 9,7€/tCO2 evitada. 
1. Una fórmula alternativa para financiar modelos de transformación hacia una economía baja en carbono con impulso a la implantación de energías renovables y medidas de eficiencia energética.

2. Una oportunidad de valorización económica de las emisiones de Gases de Efecto Invernadero (GEI) lo que incentiva la puesta en marcha en España de proyectos sostenibles que reducen o evitan la generación de $\mathrm{CO}_{2}$.

3. Un impulso para las empresas españolas, en un sector emergente y con un proceso de internacionalización en auge.

4. Una herramienta eficaz para el logro de reducciones cuantificables de emisiones de GEI y para cumplir los compromisos internacionales asumidos por España de reducción de emisiones en sectores difusos.

Las actuaciones que pueden beneficiarse de esta compraventa de tCO2 anuales evitadas son las siguientes:

1. Sector residencial: proyectos de energía térmica destinados a la reducción del consumo de combustibles fósiles en una instalación nueva o ya existente, incluyendo redes de distrito, y proyectos de eficiencia energética.

2. Sector agroganadero: proyectos de digestión anaerobia de deyecciones ganaderas (estiércoles y purines) y otros cosustratos; sustitución de combustibles fósiles por energía solar en una instalación de riego aislada nueva o ya existente.

3. Sector industrial: proyectos de eficiencia energética en el consumo de energía térmica de actividades industriales, y proyectos de captación y combustión térmica de metano en minería

4. Sector residuos: proyectos de tratamiento de residuos cuando la actividad del Proyecto Clima tiene lugar íntegramente en el vertedero o fuera de él.

5. Sector transporte: cambio de flota de turismos; cambio de flota de autobuses en transporte por carretera; cambio modal en transporte de mercancías por carretera a transporte por ferrocarril y barco.

6. Gases industriales: sustitución de HFCs por refrigerantes de menor potencial de calentamiento atmosférico, incluyendo actuaciones de sustitución de HFCs utilizados como espumantes.

Tras cuatro convocatorias anuales de Proyectos Clima, más de 200 Proyectos han sido firmados desde 2011, sumando hasta la fecha, un ahorro de emisiones de 4,3 millones de toneladas de $\mathrm{CO}^{23}{ }_{2}$. En 2016, está prevista la dotación de 20 millones de euros para esta nueva Convocatoria. El éxito de esta iniciativa impulsa la transformación del sistema productivo español hacia un modelo bajo en emisiones de gases de efecto invernadero.

\footnotetext{
${ }^{23}$ Fuente: Ministerio de Agricultura, Alimentanción y Medio Ambiente.
} 


\section{CASO PRÁCTICO DEL PROGRAMA CANAL CLIMA DE AVEBIOM. ASOCIACIÓN ESPAÑOLA DE VALORIZACIÓN ENERGÉTICA DE LA BIOMASA}

La Asociación Española de Valorización Energética de la Biomasa (AVEBIOM) se constituyó en el año 2004 con el fin de promover el desarrollo del sector de la Bioenergía en España. AVEBIOM está formada por 180 socios a nivel nacional. En esta asociación están representadas empresas de toda la cadena de valor de la bioenergía, involucradas en procesos de intercambio colaborativo.

Uno de los 49 proyectos seleccionados por la Oficina Española de Cambio Climático en la Convocatoria de proyecto Clima de 2013, de los 190 presentados, fue el "Programa de actividades de impulso de la bioenergía Canal Clima de Avebiom, Asociación Española de Valorización Energética de la Biomasa".

Este programa diseñado con un enfoque programático, en colaboración con la Corporación Tecnalia y la empresa de consultoría estratégica El Cubo Verde, persigue los siguientes objetivos:

1. Sustituir instalaciones fijas que empleen combustibles fósiles por instalaciones consumidoras de bioenergía, en instalaciones y edificios existentes o de nueva construcción, incluyendo las redes de distrito e industrias y actividades del sector agroganadero.

2. Captar y canalizar, bajo un enfoque programático, los recursos financieros necesarios, sistematizando y simplificando el acceso a los Proyectos Clima promovidos por el FES- $\mathrm{CO}_{2}$ para todas estas instalaciones, permitiendo a los asociados de AVEBIOM, acceder a este fondo destinado al desarrollo de una economía baja en carbono.

El enfoque programático permite agrupar proyectos individuales bajo un esquema común de registro y seguimiento. Facilita la presentación de proyectos y maximiza la posibilidad de ser seleccionados conforme a criterios FES- $\mathrm{CO}_{2}$, y sistematiza y simplifica la tramitación de cada instalación individual, permitiendo la incorporación de adhesiones futuras al Programa de nuevas instalaciones durante un periodo de cuatro años (hasta la fecha se han inscrito más de un centenar de instalaciones).

3. Reducir la emisión de gases de efecto invernadero en el sector de las emisiones difusas, contribuyendo a lograr los objetivos internacionalmente asumidos por España.

\subsection{Potencial del Programa Canal CLIMA en cifras}

En consumo energético, el Programa sustituye, a lo largo de sus 3 primeros años de vida, casi 2,5 millones de GJ/año de combustible fósil por bioenergía, principalmen- 
te astillas, residuos agrícolas y pellets, lo que redunda positivamente en el empleo de un recurso que además de ser neutro en carbono, es mayoritariamente local frente a nuestra dependencia exterior de combustibles fósiles en la balanza energética.

Pero además, esta iniciativa comporta beneficios ambientales y socioeconómicos: las 3 primeras actividades del Programa Canal Clima I ${ }^{24}$ (anualidades 2013, 2014 y 2015), cada una con una duración de 4 años, durante los cuales se contabilizan y verifican las emisiones reducidas a efectos de su compraventa por el FES- $\mathrm{CO}_{2}$, tienen un potencial de reducción de emisiones de $592.669 \mathrm{tCO}_{2}^{25}$ y de $240.000 \mathrm{Tep}^{26}$.

\section{Gráfica 2. Desplazamiento de combustibles fósiles por biomasa}

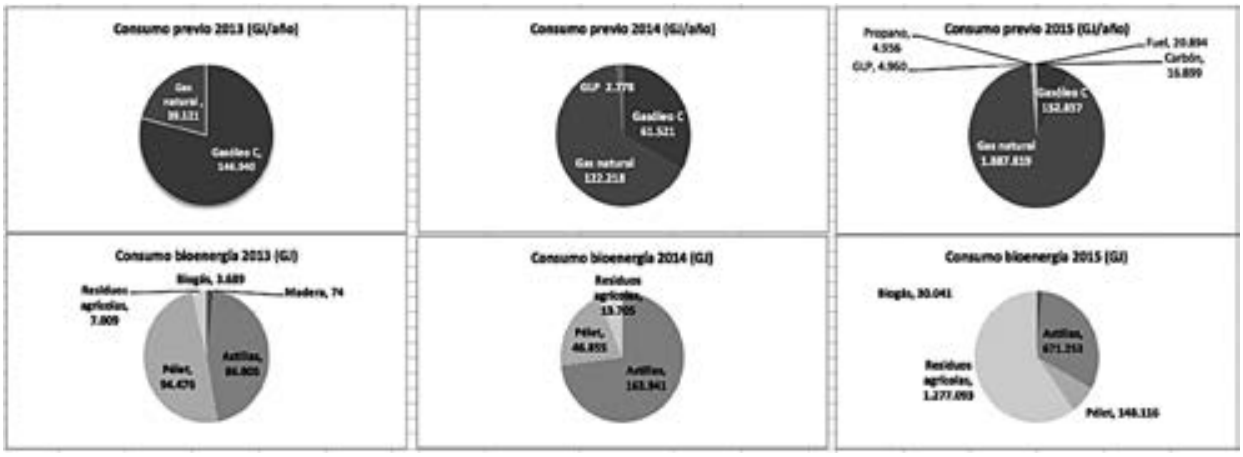

Fuente: Avebiom (Asociación Española de Valorización Energética de la Biomasa). Taller Fórmulas Alternativas de financiación (BIOFINANCE), impartido en la Feria Internacional ExpoBiomasa, Valladolid (23.09.2015) http://www.expobiomasa.com/es/taller-formulas-alternativas-de-financiacion

Además, al sustituir estos combustibles fósiles por biomasa, se minimizan los impactos que se generan en la extracción, transporte y transformación del petróleo, sin duda, superiores a los de la cadena de aprovechamiento de la biomasa. En el entorno de uso de estos combustibles tradicionales, se genera un impacto negativo en cuanto a la posible contaminación del suelo o del agua por fugas, filtraciones, roturas de depósitos, emisiones de COVs, etc. Por otra parte, los recursos forestales obtenidos de la limpia en bosques, contribuyen favorablemente a evitar la generación y propagación de incendios

${ }^{24} \mathrm{El}$ éxito de esta iniciativa entre los asociados de AVEBIOM, ha propiciado la presentación de un nuevo Programa "Programa Canal Clima II" en la Convocatoria 2015 de Proyectos Clima del FES-CO.

${ }^{25} \mathrm{El}$ cálculo de las emisiones evitadas o reducidas se hace siguiendo las metodologías aprobadas por la Oficina Española de Cambio Climático para cada actividad.

${ }^{26}$ Toneladas equivalentes de petróleo. 


\section{Gráficas 3. Beneficios ambientales y sociales del Programa Canal Clima de Avebiom}


Fuente: AVEBIOM (Asociación Española de Valorización Energética de la Biomasa). Taller Fórmulas Alternativas de financiación (BIOFINANCE), impartido en la Feria Internacional ExpoBiomasa, Valladolid (23.09.2015)

http://www.expobiomasa.com/es/taller-formulas-alternativas-de-financiacion

Como beneficios socio-económicos, el programa fomenta de manera directa el impulso de las actividades de ingeniería, mantenimiento, instalación, operación, etc. mejorando la competitividad de las empresas españolas en estos ámbitos.

Además, la promoción de este tipo de actuaciones favorece la demanda local de bienes de equipo y soluciones tecnológicas eficientes de control y optimización de la producción y consumos de demandas térmicas en industria o edificios del sector residencial, comercial y servicios. Diferentes empresas españolas son suministradores y fabricantes destacados de calderas, acumuladores y tecnología de telegestión.

Adicionalmente, el mayor consumo de biomasa frente a los combustibles fósiles favorece la competitividad y el desarrollo de toda su cadena de valor. Dado su origen local, el incremento de la demanda de biomasa, permite impulsar el uso de tecnología española en todas las etapas implicadas desde su recolección y pretratamiento y procesados, hasta su distribución final y aprovechamiento energético. Varias empresas españolas cuentan con desarrollos específicos en estos ámbitos.

El empleo de biomasa, es además una baza importante en la creación de empleo, siendo la fuente de energía que crea más puestos de trabajo por megavatio instalado (en torno a 3 puestos de trabajo por cada MWt instalado) ${ }^{27}$.

El apoyo institucional y la existencia de mecanismos adecuados de financiación como la financiación climática:

\footnotetext{
${ }^{27}$ Fuente: AVEBIOM.
} 
a) contribuye al desarrollo de este mercado cuyo objetivo son instalaciones más eficientes energéticamente y menos contaminantes, favoreciendo su consolidación;

b) generará nuevos puestos de trabajo en el sector de servicios energéticos (ingenierías, instaladoras, fabricantes, empresas mantenedoras;

c) el consumidor final podrá mejorar la rentabilidad de su instalación reduciendo la intensidad energética.

Además, la implantación de otras fuentes de energía como la biomasa contribuye al desarrollo de una actividad económica a escala local que puede favorecer especialmente a zonas rurales, generalmente más deprimidas y con niveles de empleo más bajos. Esto implica además la puesta en valor de unos recursos que afectan a toda la cadena de producto (extracción y recogida, preparación, normalización y standarización, logística, distribución, etc.

\subsection{Entrevista ${ }^{28}$ a D. Javier Díaz Presidente ${ }^{29}$ de la Asociación AVEBIOM}

A continuación se transcribe una entrevista realizada el Presidente de AVEBIOM D. Javier Díaz, en la que se describe y destacan las características de este Programa y el potencial del mismo para reducir emisiones de $\mathrm{CO}_{2}$ (mitigación) y desplazar el consumo de combustibles fósiles por biomasa.

\section{¿Cómo surgió la iniciativa de vuestro Programa CANAL CLIMA?}

En 2013, cuando nuestra organización conoció la existencia de la Convocatoria de Proyectos Clima puesta en marcha por FES- $\mathrm{CO}_{2}$ consideramos que debíamos apostar por esa vía de financiación climática. Dada la complejidad de la convocatoria, desde el principio tuvimos claro que lo más adecuado era organizar desde la asociación la presentación agrupada de las instalaciones de nuestros asociados vinculados con las instalaciones de biomasa y biogás para incrementar las posibilidades de conseguir fondos ligados a la reducción de emisiones de $\mathrm{CO}_{2}$ verificadas.

\section{¿Qué beneficios ofrece a los asociados de AVEBIOM?}

a) Las empresas asociadas a AVEBIOM adheridas a CANAL CLIMA, obtienen un retorno económico por sus instalaciones de bioenergía (incluso por las de baja potencia).

${ }^{28}$ La entrevista se realizó en la sede de AVEBIOM, el 19 de febrero de 2016 y se ha transcrito, con conformidad, a este artículo.

${ }^{29}$ Desde 2004, año de constitución de esta Asociación. 
b) Se sistematiza y simplifica el acceso los Proyectos Clima, integrando a un amplio número de promotores, que se suman al Programa bajo un mismo esquema común de registro y seguimiento.

c) AVEBIOM asume las labores de coordinación, seguimiento y verificación, ahorrando tiempo, esfuerzo y dinero a nuestros asociados.

d) Las adhesiones de nuevas actividades al Programa CANAL CLIMA, se tramitan por "vía rápida", sin necesidad de evaluación competitiva.

\section{¿Hay una tipología única de proyectos que pueden inscribirse?}

Se inscriben instalaciones de muy diversa tipología que tienen como vínculo común el uso de bioenergía como combustible. Hemos inscrito instalaciones tanto nuevas como de sustitución de combustibles fósiles, de potencia muy variada de 50 a $4.600 \mathrm{~kW}$, que usan diversos biocombustibles sólidos (pellets de madera, astilla forestal, hueso de aceituna) o biogas. Tenemos inscritas tanto instalaciones de uso doméstico como de uso industrial.

En 2015, el 9\% de las instalaciones inscritas pertenecían a redes de calor, un $30 \%$ a edificios de uso residencial, un 18\% a edificios de uso terciario, un $29 \%$ a instalaciones industriales y un $14 \%$ a instalaciones agrícolas.

¿Cuál es el potencial de reducción de emisiones de $\mathrm{CO}_{2}$ del Programa CANAL CLIMA de AVEBIOM?

AVEBIOM ha presentado un nuevo Programa Canal Clima II, que le permitirá adherir 88.068 tCO2/año hasta alcanzar 1409.088 tCO2 reducidas verificadas de 2016 a 2022.

\section{¿Qué cantidad de combustible fósil se está sustituyendo por el empleo de biomasa?}

En el Programa Canal Clima I y teniendo en cuenta las instalaciones inscritas hasta la fecha, el potencial de sustitución de combustibles fósiles por biomasa está en torno a 240.000 toneladas equivalentes de petróleo.

¿En qué porcentaje está contribuyendo AVEBIOM a conseguir los compromisos asumidos por España para reducir emisiones de $\mathrm{CO}_{2}$ en el sector de las emisiones difusas?

En 2016, las instalaciones de los dos Programas Canal Clima de AVEBIOM contribuirán a los objetivos nacionales de reducción de emisiones de $\mathrm{CO} 2$, en el sector de las emisiones difusas, en un 2,30\%. 


\section{¿Cuál es el papel que puede desempeñar la biomasa en los próximos años como energía renovable en el mix energético español?}

Sin ninguna duda, la biomasa tendrá un papel importante en el cambio de la matriz energética de nuestro país, sobre todo en lo referido a la producción térmica. Según nuestro Observatorio Nacional de Calderas de Biomasa, en este momento hay funcionando en España 160.000 equipos de biomasa con una potencia acumulada de 7.275 MW. La utilización de la biomasa como combustible tiene enormes ventajas para sustituir a los combustibles fósiles en los usos de calefacción en las viviendas, así como la sustitución en los usos industriales, o dotacionales y esto le da un protagonismo muy importante como combustible alternativo y limpio que, además, se irá incrementando según se vaya expandiendo el conocimiento de la posibilidades de los biocombustibles solidos biomásicos.

\section{FUENTES DOCUMENTALES}

\section{BOLETÍN OFICIAL DEL ESTADO}

(2006). Real Decreto 314/2006, de 17 de marzo, por el que se aprueba el Código Técnico de la Edificación. BOE núm. 74, de 28 de marzo de 2006, pp. 11816 a 11831.

(2011). Real Decreto 1494/2011, de 24 de octubre, por el que se regula el Fondo de Carbono para una Economía Sostenible. BOE núm. 270, de 9 de noviembre de 2011, pp. 116658 a 116666.

(2013) Resolución de 25 de junio de 2013, del Consejo de Administración del Instituto para la diversificación y ahorro de la Energía, publicada mediante la Resolución de 25 de septiembre de 2013 de la Secretaría de Estado de Energía, se establecieron las bases reguladoras y la convocatoria del Programa de Ayudas para la Rehabilitación Energética de Edificios Existentes del sector Residencial (uso vivienda y hotelero) (Programa PAREER) BOE núm. 235, de 1 de octubre de 2013.

(2014) Ley 18/2014, de 15 de octubre, de aprobación de medidas urgentes para el crecimiento, la competitividad y la eficiencia BOE núm. 252, de 17 de octubre de 2014, pp. 83921 a 84082.

(2015) Resolución de 28 de abril de 2015, del Instituto para la Diversificación y Ahorro de la Energía, por la que se publica la Resolución de 24 de marzo de 2015, del Consejo de Administración, por la que se establecen las bases reguladoras y convocatoria del programa de ayudas para actuaciones de eficiencia energética en PYME y en gran empresa del sector industrial. BOE número 107, de 5 de mayo de 2015, pp. 39354-39398. 


\section{DIARIO OFICIAL DE LA UNIÓN EUROPEA}

(1996) Directiva 96/61/CE, de 24 de septiembre de 1996, relativa a la prevención y al control integrados de la contaminación (IPPC) DO L 257 de 10.10.1996. (2008) Directiva 2008/1/ce del parlamento europeo y del consejo de 15 de enero de 2008 relativa a la prevención y al control integrados de la contaminación. Actualiza Directiva 96/61/CE, de 24 de septiembre de 1996, relativa a la prevención y al control integrados de la contaminación (IPPC) DO L 24/8 a 24/29 de 29.1.2008 (Versión codificada).

(2013)Decisión de la Comisión Europea 2013/162/UE de 26 de marzo de 2013, por la que se determinan las asignaciones anuales de emisiones de los Estados miembros para el período de 2013 a 2020, de conformidad con la Decisión 406/2009/CE del Parlamento Europeo y del Consejo.

\section{FUENTES DOCUMENTALES EN INTERNET}

http://ec.europa.eu/clima/policies/effort/index_en.htm

http://www.dgfc.sgpg.meh.es/

http://www.expobiomasa.com/es/taller-formulas-alternativas-de-financiacion http://www.idae.es/

http://www.gbce.es/

http://www.magrama.gob.es/ 